\title{
Retained bullet in the cervical spinal canal and the associated surgical management conundrum: case report and review of the literature
}

\author{
Joshua A. Benton ${ }^{1} \cdot$ Ralph Rahme $^{2} \cdot$ Jonathan Krystal $^{3} \cdot$ Ryan Holland $^{1} \cdot$ John K. Houten $^{4} \cdot$ Merritt D. Kinon $^{1}$
}

Received: 19 June 2020 / Revised: 5 August 2020 / Accepted: 9 August 2020

(c) International Spinal Cord Society 2020

\begin{abstract}
Introduction Gunshot wounds (GSW) to the cervical spine remain uncommon. Surgery often does not yield significant neurological improvement and the decision to utilize surgery depends on a number of factors. We describe the case of a 28 year-old male suffering a complete spinal cord injury (SCI) secondary to a bullet lodged in the cervical spinal canal. We present the unique radiological findings and review the indications for and utility of spine surgery for cervical GSW.

Case presentation The patient was a 28 year-old male involved in a motor vehicle accident immediately after sustaining a gunshot wound to the cervical spine. Neurologic exam revealed a complete SCI at the C4 level. CT scan revealed a retained bullet in the spinal canal at the $\mathrm{C} 4 / 5$ level without vascular injury or unstable vertebral fracture. He was managed nonoperatively, however, he remained ventilator dependent and ultimately expired secondary to cardiac arrest from a suspected pulmonary embolism.

Discussion We present a case of complete SCI secondary to a retained bullet in the cervical spine. These cases can be managed both operatively and nonoperatively. Given the high risk of morbidity and overall poor neurological recovery after surgical intervention for SCI secondary to GSW, physicians must understand the appropriate indications for surgical intervention. These indications include, but are not limited to, progressive neurological deficit, cerebrospinal fluid leak, spinal instability, and acute lead toxicity.
\end{abstract}

\section{Introduction}

Gunshot wounds (GSW) are the third most common cause of spinal cord injuries (SCI) in the United States, accounting for $10.4 \%$ of them [1]. Cervical spine injuries secondary to GSW are often managed nonoperatively with only a small percentage requiring surgical management [2]. GSW to the

Merritt D. Kinon

mkinon@montefiore.org

1 Department of Neurosurgery, Montefiore Medical Center/Albert Einstein College of Medicine, Bronx, NY, USA

2 Department of Surgery, Division of Neurosurgery, St. Barnabas Health System, Bronx, NY, USA

3 Department of Orthopedic Surgery, Montefiore Medical Center/ Albert Einstein College of Medicine, Bronx, NY, USA

4 Department of Neurosurgery, Donald and Barbara Zucker School of Medicine at Hofstra/Northwell and Maimonides Medical Center, Brooklyn, NY, USA cervical spine carries a higher risk of causing neurological deficits than blunt trauma with patients rarely recovering [3]. Neurological deficits include Brown-Sequard syndrome, hemiplegia, quadriplegia, and permanent ventilator dependence [4]. Spinal cord injury (SCI) can be incomplete or complete, but the overwhelming majority of penetrating cervical spine injuries result in complete injury [5]. Here we report on a rare case of complete SCI secondary to a civilian GSW to the cervical spine where the bullet is lodged in the cervical spinal canal and review the current treatment algorithm for the surgical management of this neurologically devastating injury.

\section{Case presentation}

A 28 year-old male was involved in a motor vehicle accident after being shot in the neck from behind while driving his car. He was brought to our trauma center for evaluation by EMS. On presentation, the patient was hypotensive and obtunded. All labs were normal except for toxicology 
screen positive for cocaine and marijuana. He had a gunshot entrance wound on the left lateral aspect of his neck in the mid cervical region with scant bloody ooze but no frank leakage of spinal fluid (CSF). No exit wound was seen. On neurologic exam, he did not have any spontaneous movement of or reflexes in his extremities nor motor response to deep noxious stimulation. He had a C4 sensory level and was noted to have an erection and no voluntary anal contraction.

He was intubated for airway protection and hemodynamically stabilized by the trauma surgery service. Head computed tomography (CT) and trauma imaging were all normal except for the cervical spine (Figs. 1 and 2). CT and $\mathrm{CT}$ angiogram of the cervical spine showed the bullet and bullet fragments in the middle of the spinal canal at the $\mathrm{C} 4 / 5$ level. The bullet entered via a left posterolateral approach fracturing the left C5 lamina and the inferior portion of the C5 vertebral body into the right transverse foramen and superior facet. There was no vascular injury appreciated (Fig. 3).

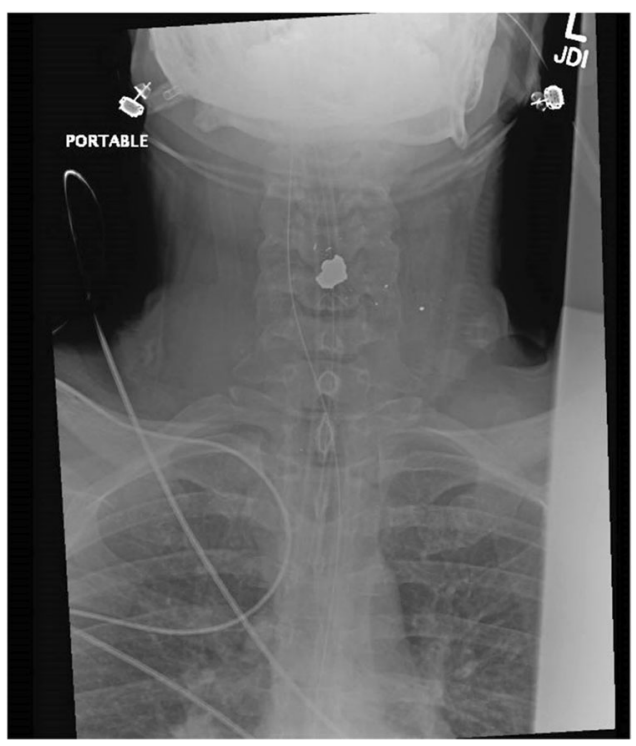

Fig. 1 Anteroposterior X-ray of the patient's cervical spine demonstrating the retained bullet within the cervical spinal canal.

Fig. 2 CT reconstruction of the patient's cervical spine demonstrating the bullet lodged within the middle of the spinal canal. a Coronal, b axial, c sagittal.

The gunshot wound was superficially debrided at the bedside and packed. It was decided to manage this injury nonoperatively since there was no active CSF leak nor spinal instability. The patient was admitted to the intensive care unit. Over the ensuing days, the patient's mental status improved, but he remained a complete SCI at the level of C4. The medical team thoroughly counseled the patient regarding his neurologic injury and prognosis for recovery. $\mathrm{He}$ expressed his understanding and desire to receive all possible care. His hospital course was complicated by failure to be weaned from the ventilator, requiring a tracheostomy on hospital day (HD) \#3 and a percutaneous endoscopic gastrostomy tube on HD \#17. He developed a heart arrhythmia requiring placement of a cardiac pacer on HD \#20 and intermittent fevers of unknown origin. On HD $\# 25$, he became acutely unresponsive and was noted to be in cardiac arrest from a presumed PE. He was resuscitated but his neurologic exam deteriorated to where he lost all brainstem reflexes. After a lengthy discussion with the family, they decided to withdraw care and the patient expired shortly thereafter.

\section{Discussion}

After medically stabilizing patients following a GSW to the cervical spine, physicians must determine the appropriateness of additional interventions. Like this case, GSW to the cervical spine is often managed nonoperatively. Of note, a bullet fragment in the spinal canal is associated with a roughly 13 -fold increase in the odds of paralysis such as in our case presentation [6]. Surgical intervention normally involves decompression and stabilization if there is a progressive neurologic deficit or spinal instability [3]. Evidence suggests surgical management may not improve neurological recovery for both incomplete and complete deficits [7]. A literature review identified two large case-series focused specifically on cervical GSW and its neurological outcomes [3,7]. Kupcha et al. determined there was no benefit to surgical decompression for incomplete or complete lesions [7]. Among patients with complete lesions

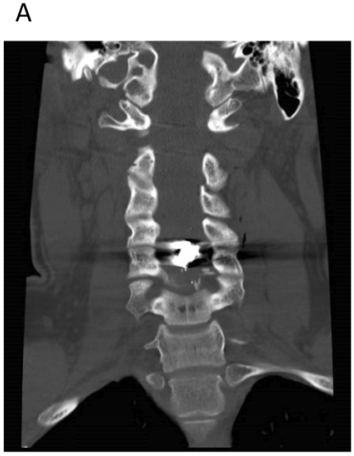

B

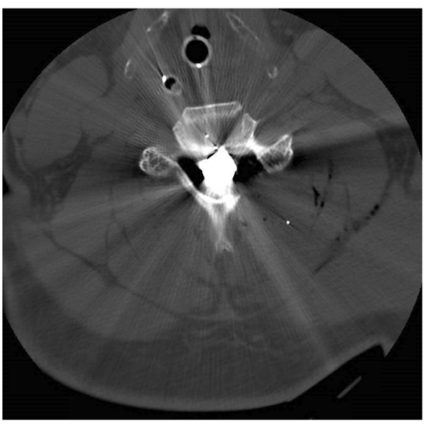

C

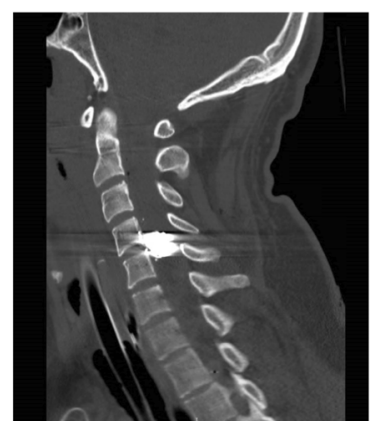


Fig. 3 Coronal reconstruction of CTA head/neck demonstrating no vascular injury.
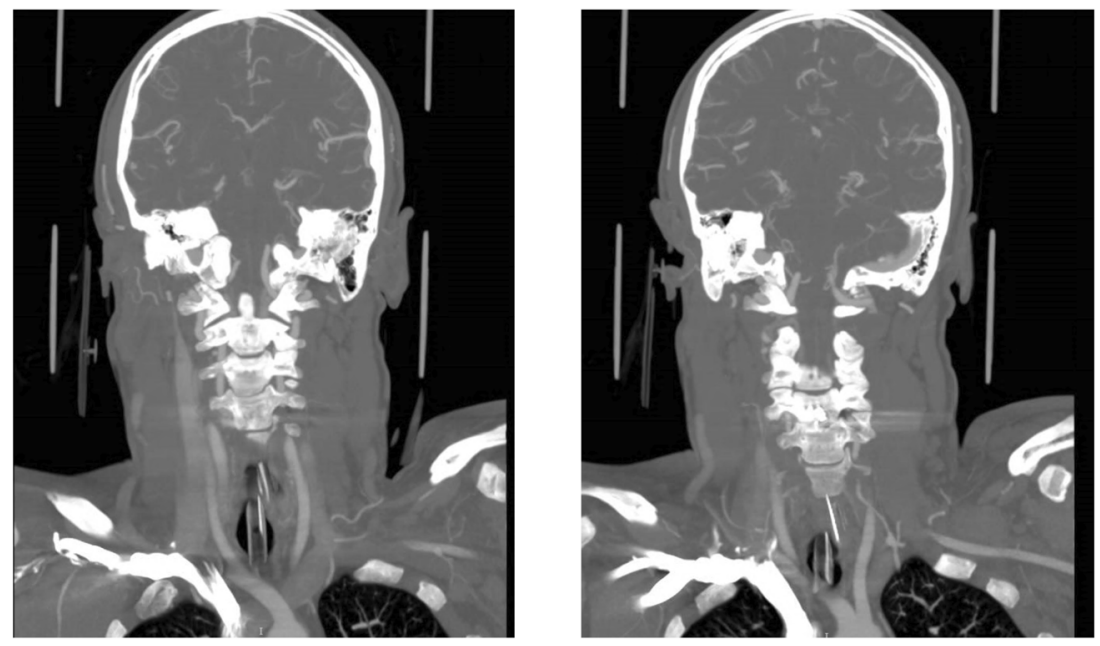

67\% improved one cervical root after surgical decompression while $64 \%$ had the same improvement without surgery; no improvement was seen among the seven patients with incomplete lesions, two of whom were managed surgically [7]. Beaty et al. did not provide a statistical comparison of neurologic recovery in surgical versus nonsurgical management; however, they recommended surgical intervention in patients with neurological decline secondary to cord compression [3]. They noted that patients with incomplete SCI demonstrated an average American Spinal Injury Association motor score improvement of 10.5 points while those with complete injuries experienced an average improvement of 5.92 points [3]. In cases managed nonoperatively several studies have attempted administering high-dose steroids given their utility in blunt trauma, but no significant clinical benefit has been achieved in these studies as it has already been shown that high-dose steroids are not beneficial and can be harmful in SCI [8]. Indications for surgical intervention include new onset progressive neurological deficit, an intracanal bullet impinging on neural elements and at risk of migration, mechanical instability, CSF leak, and evidence of acute lead intoxication [4, 9-11]. In cases requiring surgery, anterior, posterior, and combined approaches may be utilized depending on the bullet's trajectory and injury pattern to the spine and the surgeon's judgment. Lesions of the craniocervical junction have successfully been managed via a transoral approach while laminectomy and the other standard approaches may be more appropriate for the subaxial cervical spine [4, 12].

Tetanus prophylaxis is recommended for all patients if their last immunization date is unknown while antibiotic prophylaxis for $48-72 \mathrm{~h}$ may reduce infection secondary to airway or esophageal perforation [4]. Cervical collars should be utilized until deemed unnecessary after a thorough spine evaluation. Along with correction of and continued monitoring for hemodynamic instability, immobile patients should be turned regularly, perform breathing exercises to prevent respiratory complications, receive physical and mechanical deep venous thrombosis prophylaxis. and engage in regular physical rehabilitation exercises [13].

If surgery is utilized, its timing may impact the rates of certain sequelae of GSW to the spine. When there is concern for infection and a need for wound debridement, surgery performed within two weeks of injury has been associated with lower rates of infection and arachnoiditis in the lumbar spine [14]. Although the lumbar spine is associated with the risk of abdominal viscus perforation and contamination with intestinal contents, timing of surgery in the cervical spine may also impact these outcomes and further research is required on this issue. Retained bullets-particularly those in contact with CSF or synovial fluid-may cause lead intoxication in the future; early surgical removal may prevent the development of this sequela $[4,15]$. Another late sequelae of traumatic SCI is progressive neurological loss due to spinal cord tethering, myelomalacia, or syringomyelia [16]. Surgical intervention, such as spinal detethering and cyst shunting when indicated, can successfully arrest these progressive deficits [16].

The acute nature of the individual's SCI, resulting complete deficit, and lack of spinal instability and cerebrospinal fluid leak allowed us to manage this patient nonoperatively. In patients with progressive onset of a deficit, intervention would be warranted. When considering surgical management, surgeons must weigh the risks associated with their intervention. Surgical intervention increases the risk of morbidity for a patient population that already experiences a morbidity rate that may exceed $90 \%$ [7]. In the peri- and postoperative period, complications include urinary tract infection, pneumonia, and wound infection [7]. Surgical procedures, and laminectomy in particular, also carry long-term risks. Laminectomy within 
two weeks of sustaining a GSW has been associated with a higher incidence of cerebrospinal-cutaneous fistulae. Long term, it may destabilize the spine, leading to deformity [4].

A GSW to the cervical spine carries significant morbidity. Here we present a unique case where the bullet was lodged within the middle of the spinal canal and caused a complete SCI. Management of civilian GSW to the cervical spinal cord can be performed in a variety of ways depending on the degree of neurologic injury and the stability of the spine, as well as other associated injuries. Given the high risk of morbidity and overall poor neurological recovery after surgical intervention for SCI secondary to GSW, physicians must understand the appropriate indications for surgical intervention.

\section{Compliance with ethical standards}

Conflict of interest The authors declare that they have no conflict of interest.

Publisher's note Springer Nature remains neutral with regard to jurisdictional claims in published maps and institutional affiliations.

\section{References}

1. Chen Y, Tang Y, Vogel LC, Devivo MJ. Causes of spinal cord injury. Top Spinal Cord Inj Rehabil. 2013;19:1-8.

2. Medzon R, Rothenhaus T, Bono CM, Grindlinger G, Rathlev NK. Stability of cervical spine fractures after gunshot wounds to the head and neck. Spine. 2005;30:2274-9.
3. Beaty N, Slavin J, Diaz C, Zeleznick K, Ibrahimi D, Sansur CA. Cervical spine injury from gunshot wounds. J Neurosurg Spine. 2014;21:442-9.

4. Bono CM, Heary RF. Gunshot wounds to the spine. Spine J. 2004;4:230-40.

5. Young JS, Burns PE, Bowen AM. Spinal cord injury statistics: experience of the regional spinal cord injury systems: Good Samaritan Medical Center. Phoenix. 1982;1-152.

6. Chittiboina P, Banerjee AD, Zhang S, Caldito G, Nanda A, Willis BK. How bullet trajectory affects outcomes of civilian gunshot injury to the spine. J Clin Neurosci. 2011;18:1630-3.

7. Kupcha PC, An HS, Cotler JM. Gunshot wounds to the cervical spine. Spine. 1990;15:1058-63.

8. Sidhu GS, Ghag A, Prokuski V, Vaccaro AR, Radcliff KE. Civilian gunshot injuries of the spinal cord: a systematic review of the current literature. Clin Orthop Relat Res ${ }^{\circledR}$. 2013;471:3945-55.

9. Grogan DP, Bucholz RW. Acute lead intoxication from a bullet in an intervertebral disc space. A case report. JBJS. 1981;63:1180-2.

10. Wigle RL. Treatment of asymptomatic gunshot injuries to the spine. Am Surg. 1989;55:591-5.

11. de Barros Filho T, Cristante A, Marcon R, Ono A, Bilhar R. Gunshot injuries in the spine. Spinal Cord. 2014;52:504-10.

12. Maniker AH, Gropper MR, Hunt CD. Transoral gunshot wounds to the atlanto-axial complex: report of five cases. J Trauma. 1994;37:858-61.

13. El Masri WS. Traumatic spinal injury and spinal cord injury: point for active physiological conservative management as compared to surgical management. Spinal Cord Ser Cases. 2018;4:14.

14. Cybulski GR, Stone JL, Kant R. Outcome of laminectomy for civilian gunshot injuries of the terminal spinal cord and cauda equina: review of 88 cases. Neurosurgery. 1989;24:392-7.

15. John BE, Boatright D. Lead toxicity from gunshot wound. South Med J. 1999;92:223-4.

16. Falci SP, Indeck C, Lammertse DP. Posttraumatic spinal cord tethering syringomyelia: surgical treat long-term outcome. J Neurosurg Spine. 2009;11:445. 